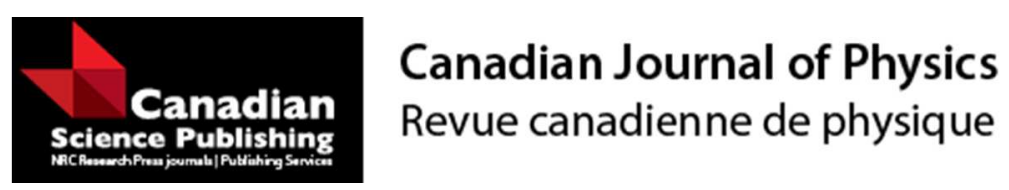

\title{
Thermodynamic Properties of Graphene Using the Static Fluctuation Approximation (SFA)
}

\begin{tabular}{|r|l|}
\hline Journal: & Canadian Journal of Physics \\
\hline Manuscript ID & cjp-2016-0310.R3 \\
\hline Manuscript Type: & Article \\
\hline Date Submitted by the Author: & 20 -Oct-2016 \\
\hline Complete List of Authors: & $\begin{array}{l}\text { Hawamdeh, Mustafa; Al-Balqa' Applied University, Department of Physics } \\
\text { Al-Sugheir, Mohamed; The Hashemite University, Physics Department } \\
\text { Sandouqa, Ayman; Al-Balqa Applied University, } \\
\text { Ghassib, Humam; The University of Jordan, Physics Department }\end{array}$ \\
\hline Keyword: & $\begin{array}{l}\text { Two-dimensional System, graphene nanosystems, nonextensive statistical } \\
\text { mechanics, static fluctuation approximation, Thermodynamic Properties }\end{array}$ \\
\hline
\end{tabular}


Thermodynamic Properties of Graphene Using the Static Fluctuation Approximation (SFA)

Mustafa M. Hawamdeh ${ }^{1}$, Mohamed K. Al-Sugheir ${ }^{2}$, Ayman S.Sandouqa ${ }^{1}$, and Humam B. Ghassib $^{3}$

${ }^{1}$ Department of Physics, Faculty of Engineering Technology, Al-Balqa' Applied University, Amman, Jordan

${ }^{2}$ Department of Physics, The Hashemite University, Zarqa, Jordan

${ }^{3}$ Department of Physics, Faculty of Science, The University of Jordan, Amman, Jordan

Section: Condensed Matter Physics

Suggested Running Head: Thermodynamic Properties of Graphene Using SFA

Corresponding author:

Prof. Dr. Mohamed K. Al-Sugheir

Department of Physics

The Hashemite University

Zarqa, Jordan

E-mail:msugh@hu.edu.jo 


\begin{abstract}
The thermodynamic properties of two-dimensional graphene nanosystems are investigated using the static fluctuation approximation (SFA). These properties are analyzed using both extensive and nonextensive statistical mechanics. It is found that these properties are less sensitive to the temperature when using nonextensive - in contrast to extensive statistical mechanics. It is also noted that the mean internal energy and the specific heat behave as a power-law, $T^{\alpha}$, at $T<8 \mathrm{eV}$; whereas they go to the classical limit for the twodimensional ideal gas at $T>8 \mathrm{eV}$.

The results are presented in a set of figures and one table. The roles played by the number of particles and the entropy parameter $\mathrm{q}$ are underlined. Whenever possible, comparisons are made to previous studies. It is concluded that Boltzmann-Gibbs statistics is not valid for some cases, and that SFA results are in good agreement with those obtained within other formalisms.
\end{abstract}

Keywords: Two-dimensional graphene nanosystems; nonextensive statistical mechanics; static fluctuation approximation.

PACS numbers: 65.80.Ck, 68.65.Pq 


\section{Introduction}

Graphene is a 2D-honeycomb lattice of carbon atoms. Its experimental realization has opened up new horizons in condensed-matter physics and material science. It is becoming increasingly important in electronics and material science for its outstanding electrical, thermal and mechanical properties. Graphene possesses four valence electrons for each carbon atom. Three of these (s-electrons) form tight bonds with the neighboring atoms in the plane and do not play a part in the conduction phenomenon. The fourth (p-electron) may move freely in the positive-ion lattice. Unique electronic properties make graphene substantially different from the conventional two-dimensional electron gas (2DEG).

Theoretically, various models have been adopted for studying this system. The electronic structure of graphene can be reasonably described using a simple tight-binding Hamiltonian $[1,2]$. The spectrum is derived by solving the Schrödinger equation analytically for the electronic energy dispersion and related eigenstates [1]. Ezawa [2] studied the electronic properties of graphene derivatives which have closed edges. They are finite-length graphene nanoribbons and graphene nanodisks. No metallic states are found in finite-length zigzag nanoribbons; but all infinite-length zigzag nanoribbons are metallic. Also, no metallic states are found in hexagonal, parallelogrammic and trigonal nanodisks with zigzag or armchair edges, except trigonal zigzag nanodisks. The degeneracy of the metallic states in trigonal zigzag nanodisks can arbitrarily be designed by changing the size. Grushin et al. [3] proposed a phenomenological model that included the linear behavior of the scattering rate at low frequencies, and showed that it could explain qualitatively optical-conductivity experiments of doped graphene in the infrared regime. Strictly speaking, graphene should be described by the relativistic Dirac equation, rather than the non-relativistic Schrödinger equation, with an effective mass, because the electronic group velocity - estimated at the Dirac points - is quite high $\sim 1 \times 10^{6} \mathrm{~m} / \mathrm{s}[4,5]$.

Experimentally, transport and thermodynamic properties have been studied. An unusual quantum Hall effect (QHE) was discovered in high-quality graphene samples [6]. In contrast to conventional $2 \mathrm{D}$ systems, the observed quantization condition in graphene is described by half-integer, rather than integer, values. Markin et al. [7] studied the temperature dependence of the heat capacities of disordered graphite-like nanostructures prepared by the thermobaric treatment of fullerite $\mathrm{C}_{60}$ in the temperature-interval from 7 to $610-650 \mathrm{~K}$. Two samples were prepared by treating the fcc fullerite $\mathrm{C}_{60}$ under high pressure ( 2 and $8 \mathrm{GPa}$ ) and temperature $(1373 \mathrm{~K})$, for $2000 \mathrm{~s}$. Then the pressure and temperature were abruptly decreased 
to $0.1 \mathrm{MPa}$ and $298 \mathrm{~K}$ to produce thermodynamically metastable structures. It was found that the heat capacity of these two samples depended on $T$ to the power 2.5 and 2.7.

Several previous studies were interested in the thermodynamic properties of twodimensional graphene. Wright et al. [8] used the electronic states in the tight-binding approximation to calculate the thermal and magnetic properties of graphene nanoribbons under zero and finite magnetic fields. Nasir et al. [9] studied the thermodynamic properties of a monolayer graphene system and compared the results to those of the conventional 2DEG. They found the system to be less sensitive to the temperature and more sensitive to the magnetic field, as compared to 2DEG. Li et al. [10] calculated the chemical potential, total energy and specific heat as functions of the temperature, carrier concentration and the effective mass at low temperatures with relativistic effects. They evaluated and discussed the density distribution of massive Dirac fermions as functions of the effective mass and the carrier density, which could be varied in doped or gated graphene systems. They distinguished between two cases: with and without relativistic effects. For massless fermions, the total kinetic energy was equal to a constant $\sim \mathrm{n}^{3 / 2}$ for a given density $\mathrm{n}$; and the specific heat maintained its linear behavior with temperature. Yi et al. [11] studied the low-energy electronic energy structure of a graphene strip near band-crossing points and made use of the finite width effect in the low-temperature heat capacity, $C_{e l}(T)$. They found that the lowenergy electronic structure depended strongly on the strip width and the relative strength of various spin-charge coupling mechanisms, and that the overall behavior of $C_{e l}(T)$ was similar to that of a two-level system. Here, as shown later, we find that the specific heat obeys a power-law behavior $\propto T^{\alpha}$, with $\alpha=2.0 \sim 3.5$ in the metallic region at extremely low temperatures.

In this work, the thermodynamic properties of two-dimensional graphene nanosystems are investigated. The properties considered are the internal energy, the occupation number, the specific heat capacity and entropy. We shall use the static fluctuation approximation (SFA) [12-15]. SFA is a relatively simple approach in which the square of the local-field operator is replaced with its mean value. The physical implication is that the true quantum-mechanical spectrum of this operator is replaced with a distribution around its expectation value. This approximation has been used successfully to study the proton model of ferroelectrics with tunneling [13], neutral many-bosonic systems [14], neutral many-fermionic systems [15, 16], spin-polarized systems [17, 18], hot nuclear matter [19] and trapped Bose gases [20]. Here, SFA will be invoked to study the thermodynamic properties of graphene nanosystems. The 
corresponding algorithm, with Boltzmann-Gibbs statistical mechanics, is based on nonextensive statistical mechanics. Only nonrelativistic effects are taken into account; a relativistic treatment demands a drastic modification of SFA, which will be attempted in a future work. Thus, the present calculations may be regarded as a first step in studying graphene within the framework of SFA.

We have considered a nanosystem of carbon atoms of graphene strips, each atom contributing one free electron of mass $m_{e}$. The electrons are confined to a finite twodimensional 'box'. Nonextensive statistical mechanics will be invoked to calculate the thermodynamic properties of this system [21-23]. The basic principles of thermodynamics and statistical mechanics for small systems were formulated some time ago [24]. Since then there has been a spate of studies. For example, Nassimi and Parsafar [25] used scaling methods to render nonextensive thermodynamic functions of such systems extensive, and vice versa. Employing extensive molecular dynamic simulations, Mohazzabi and Mansoori [21] investigated the extensivity of the internal energy and entropy, as well as the intensivity of the temperature and pressure, in small atomic systems consisting of less than 1000 argon-like particles; interacting through the Lennard-Jones potential. They found that, in small systems, contrary to macroscopic systems, the internal energy and entropy are nonextensive; whereas the temperature and pressure are nonintensive.

The rest of the paper is organized as follows. The model of our system is described in Section 2. Section 3 summarizes the basic principles and techniques of SFA for nanosystems using nonextensive statistical mechanics. The corresponding closed set of nonlinear equations, which is an essential feature of SFA, is the subject of Section 4. The thermodynamic properties of nanosystems are written down in Section 5. The results are presented and discussed in Section 6. Finally, in Section 7, the paper ends with some concluding remarks.

\section{Graphene System}

Graphene can be modeled by a neutral system of $\mathrm{N}$ interacting electrons moving on a smooth finite 2D surface [say, in the xy-plane], with a uniform positive background consisting of $\mathrm{N}$ carbon ions.

From basic quantum mechanics, the wavefunction of a single electron moving in a finite two-dimensional 'box' with sides of lengths $l_{1}, l_{2}$ is 


$$
\psi(x, y)=\sqrt{\frac{2}{\ell_{1} \ell_{2}}} \sin k_{x} x \sin k_{y} y .
$$

The periodic boundary conditions determine the allowed wavenumbers as

$$
k_{x}=\frac{n \pi}{\ell_{1}} \quad \text { and } \quad k_{y}=\frac{m \pi}{\ell_{2}},
$$

$n$ and $m$ being positive integers.

The eigenvalues $\varepsilon_{n m}$ of the single-electron energy are given by

$$
\varepsilon_{n m}=\frac{\hbar \pi^{2}}{2 m_{e}}\left\{\frac{n^{2}}{\ell_{1}^{2}}+\frac{m^{2}}{\ell_{2}^{2}}\right\}
$$

$m_{e}$ being the electron mass.

The total Hamiltonian describing the system can be written as the sum of three terms:

$$
H=H_{0}+H_{e l-c}+H_{1}
$$

where $H_{0}$ is the single-electron Hamiltonian with eigenvalues $\varepsilon_{n m}, H_{e l-c}$ is the electron-carbon ions interaction, and $H_{1}$ is the electron-electron interaction.

The interaction energy between an electron and a carbon ion is given by [26]

$$
H_{e l-c}=-e \sum_{i=1}^{N} \int_{0}^{\ell_{1}} \int_{0}^{\ell_{2}} d x d y \frac{n(x, y) e^{-\eta \sqrt{\left(x-x_{i}\right)^{2}+\left(y-y_{i}\right)^{2}}}}{\sqrt{\left(x-x_{i}\right)^{2}+\left(y-y_{i}\right)^{2}}} .
$$

Here, $\left(x_{i}, y_{i}\right)$ is the position of the $i^{\text {th }}$ electron. The exponential convergence factor is inserted to ensure the convergence of the integral; $\eta$ will eventually be allowed to vanish because of the long-range nature of the Coulomb interaction. The electron-carbon ions interaction $H_{e l-c}$ is a one-particle operator since it acts on each electron individually. The positive ions are much heavier than the electrons; so the ionic motion can be neglected to a first approximation (the well-known adiabatic or Born-Oppenheimer approximation). In our work, graphene was modeled by a system of $\mathrm{N}$ interacting electrons placed on a smooth finite $2 \mathrm{D}$ surface, with a uniform positive background. For such a uniform distribution, the ion density $n(x, y)=$ $N / \ell_{1} \ell_{2}$ [26]. The only dynamic variables are those pertaining to electrons, because the carbon ions are localized. Thus, $H_{e l-c}$ is a c-number [26]. This term is included in the effective chemical potential of graphene; it causes an enhancement of the Fermi degeneracy temperature $T_{F}$ of the system. All interesting physical effects are thereby contained in the electron-electron interaction $\mathrm{H}_{1}$.

In second quantization, the Hamiltonian $\left(H=H_{0}+H_{e l-c}+H_{1}\right)$ for $\mathrm{N}$ interacting fermions in a 'box' is given by 


$$
\begin{aligned}
& \widehat{H}=\sum_{n m} \varepsilon_{n m} \hat{a}_{n m}^{+} \hat{a}_{n m} \\
&+\sum_{n_{1} m_{1}} \sum_{n_{2} m_{2}} \sum_{n_{3} m_{3}} \sum_{n_{4} m_{4}} \frac{16}{2 \ell_{1}{ }^{2} \ell_{2}^{2}} \int_{0}^{\ell_{1}} \int_{0}^{\ell_{2}} \int_{0}^{\ell_{2}} \int_{0}^{\ell_{1}} \sin \frac{n_{1} \pi x_{1}}{\ell_{1}} \sin \frac{m_{1} \pi y_{1}}{\ell_{2}} \sin \frac{n_{2} \pi x_{2}}{\ell_{1}} \sin \frac{m_{2} \pi y_{2}}{\ell_{2}} \\
& \quad \times \mathrm{V}\left(x_{1}, y_{1}, x_{2}, y_{2}\right) \sin \frac{n_{3} \pi x_{2}}{\ell_{1}} \sin \frac{m_{3} \pi y_{2}}{\ell_{2}} \sin \frac{n_{4} \pi x_{1}}{\ell_{1}} \sin \frac{m_{4} \pi y_{1}}{\ell_{2}} \\
& \hat{a}_{n_{1} m_{1}}^{+} \hat{a}_{n_{2} m_{2}}^{+} \hat{a}_{n_{3} m_{3}} \hat{a}_{n_{4} m_{4}} d x_{1} d y_{1} d y_{2} d x_{2},
\end{aligned}
$$

$\hat{a}_{n m}^{+}, \hat{a}_{n m}$ being the creation and annihilation operators for an electron in the state $|n m\rangle$.

The grand canonical Hamiltonian of this system is

$$
\begin{aligned}
\widehat{H} & =\sum_{n m}\left(\varepsilon_{n m}-\mu\right) \hat{a}_{n m}^{+} \hat{a}_{n m} \\
& +\sum_{n_{1} m_{1} n_{2} m_{2} n_{3} m_{3} n_{4} m_{4}} V_{n_{1} m_{1} n_{2} m_{2} n_{3} m_{3} n_{4} m_{4}} \hat{a}_{n_{1} m_{1}}^{+} \hat{a}_{n_{2} m_{2}}^{+} \hat{a}_{n_{3} m_{3}} \hat{a}_{n_{4} m_{4}},
\end{aligned}
$$

where $V_{n_{1} m_{1} n_{2} m_{2} n_{3} m_{3} n_{4} m_{4}}$ is the interaction matrix element, defined as

$$
V_{n_{1} m_{1} n_{2} m_{2} n_{3} m_{3} n_{4} m_{4}}=\frac{1}{2} \sum_{n_{1} m_{1} n_{2} m_{2} n_{3} m_{3} n_{4} m_{4}}\left\langle n_{1} m_{1} n_{2} m_{2}|V| \overrightarrow{r_{1}}-\overrightarrow{r_{2}}|| n_{3} m_{3} n_{4} m_{4}\right\rangle,
$$

$\mu$ being the 'effective' chemical potential of graphene.

\section{SFA Formalism}

For a specific Hamiltonian $\widehat{H}$, the Heisenberg representation of a creation operator $\hat{a}_{n m}^{+}$ is given by

$$
\hat{a}_{n m}^{+}(\tau)=e^{\tau \widehat{H}} \hat{a}_{n m}^{+}(0) e^{-\tau \widehat{H}},
$$

where $\tau=i t$. The equation of motion of the creation operator in this representation is

$$
\frac{d \hat{a}_{n m}^{+}(\tau)}{d \tau}=\left[\widehat{H}, \hat{a}_{n m}^{+}(\tau)\right] .
$$

Here, $n$ and $m$ are indices denoting the complete set of compatible quantum numbers describing a specific state.

In SFA [12-15], the total Hamiltonian can be expressed as a linear combination of the local-field operator $\hat{E}_{n m}$ and the number-of-particles operator $\hat{n}_{n m}$ :

$$
\widehat{H}=\sum_{n m} \hat{E}_{n m} \hat{n}_{n m},
$$

where $\hat{n}_{n m}=\hat{a}_{n m}^{+} \hat{a}_{n m}$, and $\hat{E}_{n m}$ is to be found from the equation of motion for the operator in the Heisenberg picture: 


$$
\frac{d \hat{a}_{n m}^{+}(\tau)}{d \tau}=\left[\widehat{H}, \hat{a}_{n m}^{+}(\tau)\right]=\hat{E}_{n m} \hat{a}_{n m}^{+}(\tau)
$$

So

$$
\widehat{E}_{n m}=\left\{\hat{a}_{n m},\left[\widehat{H}, \hat{a}_{n m}^{+}(\tau)\right]\right\} .
$$

$\widehat{E}_{n m}$ is assumed to be hermitian; it commutes with the creation and annihilation operators according to the well-known relations for a Fermi system:

$$
\left\{\hat{a}_{k l}^{+}, \hat{a}_{n m}\right\}=\delta_{k n} \delta_{l m} ;\left\{\hat{a}_{k l}^{+}, \hat{a}_{n m}^{+}\right\}=0 .
$$

Then $\hat{E}_{n m}$ can be calculated as follows:

$$
\begin{aligned}
{\left[\widehat{H}, \hat{a}_{n m}^{+}\right] } & =\varepsilon_{n m} \hat{a}_{n m}^{+}+\sum_{n_{1} m_{1}} \sum_{n_{2} m_{2}} \sum_{n_{3} m_{3}} V_{n_{1} m_{1} n_{2} m_{2} n_{3} m_{3} n m} \hat{a}_{n_{1} m_{1}}^{+} \hat{a}_{n_{2} m_{2}}^{+} \hat{a}_{n_{3} m_{3}} \\
& -\sum_{n_{1} m_{1}} \sum_{n_{2} m_{2}} \sum_{n_{4} m_{4}} V_{n_{1} m_{1} n_{2} m_{2} n m n_{4} m_{4}} \hat{a}_{n_{1} m_{1}}^{+} \hat{a}_{n_{2} m_{2}}^{+} \hat{a}_{n_{4} m_{4}} \cdot
\end{aligned}
$$

Using Eq. (12), we have

$$
\begin{aligned}
\hat{E}_{n m}=\varepsilon_{n m}+ & \sum_{n_{1} m_{1} n_{3} m_{3}} V_{n_{1} m_{1} n m n_{3} m_{3} n m} \hat{a}_{n_{1} m_{1}}^{+} \hat{a}_{n_{3} m_{3}} \\
& -\sum_{n_{2} m_{2} n_{3} m_{3}} V_{n m n_{2} m_{2} n_{3} m_{3} n m} \hat{a}_{n_{2} m_{2}}^{+} \hat{a}_{n_{3} m_{3}} \\
& -\sum_{n_{1} m_{1} n_{4} m_{4}} V_{n_{1} m_{1} n m n m n_{4} m_{4}} \hat{a}_{n_{1} m_{1}}^{+} \hat{a}_{n_{4} m_{4}} \\
& +\sum_{n_{2} m_{2} n_{4} m_{4}} V_{n m n_{2} m_{2} n m n_{4} m_{4}} \hat{a}_{n_{2} m_{2}}^{+} \hat{a}_{n_{4} m_{4}}
\end{aligned}
$$

Since

$$
\begin{gathered}
\left\langle n m\left|\hat{E}_{k l}\right| n m\right\rangle=\delta_{n k} \delta_{m l}, \\
\left\langle\hat{E}_{n m}\right\rangle=\varepsilon_{n m}+\sum_{i j}\left\{V_{i j n m i j n m}-V_{n m i j i j n m}-V_{i j n m n m i j}+V_{n m i j n m i j}\right\}\left\langle n_{i j}\right\rangle .
\end{gathered}
$$

From symmetry, the two positive terms are equal; so are the two negative terms. Thus,

$$
\left\langle\widehat{E}_{n m}\right\rangle=\varepsilon_{n m}+\sum_{i j}\left\{2 V_{i j n m i j n m}-2 V_{n m i j i j n m}\right\}\left\langle n_{i j}\right\rangle ;
$$

or

$$
\left\langle\widehat{E}_{n m}\right\rangle=\varepsilon_{n m}+\sum_{i j} C_{i n j m}\left\langle n_{i j}\right\rangle
$$

with

$$
C_{n i m j} \equiv 2 V_{i j n m i j n m}-2 V_{n m i j i j n m}
$$


The local-field operator $\widehat{E}_{n m}$ can be written as:

$$
\widehat{E}_{n m}=\left\langle\widehat{E}_{n m}\right\rangle+\Delta \hat{E}_{n m},
$$

where $\left\langle\widehat{E}_{n m}\right\rangle$ is the mean energy of the system, and $\Delta \hat{E}_{n m}$ is the corresponding deviation $[13,14]$ :

$$
\Delta \widehat{E}_{n m}=\sum_{i \neq j} C_{n i m j} \Delta n_{i j}
$$

In SFA [13], the square of the quadratic-fluctuations operator is replaced with its mean value:

$$
\left(\Delta \hat{E}_{n m}\right)^{2} \cong\left\langle\left(\Delta \hat{E}_{n m}\right)^{2}\right\rangle=\varphi_{n m}^{2}
$$

\section{Closed Set of Nonlinear Equations for Finite Systems in SFA}

As already mentioned, SFA has been extensively used for investigating infinite systems [12-15]. In this work, SFA is applied to a finite nanosystem. Therefore, instead of the usual extensive statistical mechanics, Tsallis nonextensive statistical mechanics [3] will be used. The corresponding solution of the Heisenberg equation of motion within this 'generalized' statistical mechanics and SFA can be written as

$$
\hat{a}_{n m}^{+}(\tau)=\hat{a}_{n m}^{+}(0) e_{q}^{\hat{E}_{n m} \tau}
$$

where

$$
e_{q}^{x}=[1+(1-q) x]^{\frac{1}{1-q}}
$$

The desired generating equation for nonextensive systems can be obtained from the following expression for the expectation value:

$$
\begin{aligned}
\langle\hat{A}(\beta) C\rangle_{q} & =\operatorname{Tr}\left(\rho^{q} \hat{A}(\beta) C\right) \\
& =\operatorname{Tr}\left(\left[e_{q}^{-\beta \hat{E}_{n m}}\right]^{q}\left[e_{q}^{-\beta \hat{E}_{n m}}\right]^{-1} \hat{A}\left[e_{q}^{-\beta \hat{E}_{n m}}\right] C\right),
\end{aligned}
$$

the probability function $\rho$ being defined as

$$
\rho=e_{q}^{-\beta \hat{E}_{n m}}=\left[1-(1-q) \beta \hat{E}_{n m}\right]^{\frac{1}{1-q}}
$$

Here and below, we shall replace $\tau$ with $\beta \equiv 1 / k_{B} T, k_{B}$ being Boltzmann's constant and $T$ the absolute temperature; this is common in statistical mechanics [27].

Equation (26) can be derived according to the following steps: 
The local-field operator is now given by [28]

$$
\widehat{E}_{n m}=\frac{1-e^{-(1-q) \beta \widehat{W}}}{(1-q) \beta} .
$$

Based on this transformation,

$$
e_{q}^{-\beta \hat{E}_{n m}}=e^{-\beta \widehat{W}}
$$

Use Eq. (29) to rewrite (26) in terms of the $\widehat{W}$ operator:

$$
\langle\hat{A}(\beta) C\rangle_{q}=\operatorname{Tr}\left(\left[e^{-\beta \widehat{W}}\right]^{q}\left[e^{-\beta \widehat{W}}\right]^{-1} \hat{A}\left[e^{-\beta \widehat{W}}\right] C\right) .
$$

Since the trace is cyclic, Eq. (30) can be written as

$$
\langle\hat{A}(\beta) C\rangle_{q}=\operatorname{Tr}\left(\text { one }\left[e^{-\beta \widehat{W}}\right] C\left[e^{-\beta \widehat{W}}\right]^{q}\left[e^{-\beta \widehat{W}}\right]^{-1} \hat{A}\right) .
$$

Writing one $\equiv e^{-q \beta \widehat{W}} e^{q \beta \widehat{W}}$, then

$$
\begin{aligned}
\langle\hat{A}(\beta) C\rangle_{q} & =\operatorname{Tr}\left[\mathrm{e}^{-q \beta \widehat{W}} \mathrm{e}^{q \beta \widehat{W}} \mathrm{e}^{-\beta \widehat{W}} C e^{-q \beta \widehat{W}} \mathrm{e}^{\beta \widehat{W}} \hat{A}\right] \\
& =\operatorname{Tr}\left[\mathrm{e}^{-q \beta \widehat{W}} \mathrm{e}^{(q-1) \beta \widehat{W}} C e^{-(q-1) \beta \widehat{W}} \hat{A}\right] \\
& =\langle\hat{C}((q-1) \beta) \hat{A}\rangle_{q} \\
& =\left\langle\left[e_{q}^{-\beta \hat{E}_{n m}}\right]^{q}\left[e_{q}^{-\beta \hat{E}_{n m}}\right]^{-(q-1)} \hat{C}\left[e_{q}^{-\beta \hat{E}_{n m}}\right]^{(q-1)} \hat{A}\right\rangle_{q} .
\end{aligned}
$$

For $q=1$, we go back to the extensive form [14], as we should.

From Eq. (33), the generating equation for nonextensive systems can be derived as follows:

Let

and

$$
\begin{aligned}
\hat{A}(\beta) & =\hat{a}_{n m}^{+}(\beta), \\
\hat{C} & =\hat{a}_{n m} \hat{B},
\end{aligned}
$$

$\widehat{\mathrm{B}}$ being an arbitrary operator.

Equation (33) becomes

$$
\left\langle\hat{a}_{n m}^{+}(\beta) \hat{a}_{n m} \hat{B}\right\rangle_{q}=\left\langle\hat{a}_{n m}((q-1) \beta) \hat{B} \hat{a}_{n m}^{+}\right\rangle_{q} .
$$

This can be rewritten in the form

$$
\left\langle\left(e_{q}^{-\beta \hat{E}_{n m}}\right)^{-1} \hat{a}_{n m}^{+} \hat{a}_{n m} \hat{B}\right\rangle_{q}=\left\langle\left(e_{q}^{-(q-1) \beta \hat{E}_{n m}}\right) \hat{a}_{n m} \hat{B} \hat{a}_{n m}^{+}\right\rangle_{q},
$$

where the local-field operator commutes with the creation and annihilation operators.

Choose $\hat{B}$ to commute with the local-field operator as well as with the creation and annihilation operators. Then, Eq. (36) becomes

$$
\left\langle\left(e_{q}^{-\beta \hat{E}_{n m}}\right)^{-1} \hat{n}_{n m} \hat{B}\right\rangle_{q}=\left\langle\left(\left(e_{q}^{-(q-1) \beta \hat{E}_{n m}}\right)\right)\left(1-\hat{n}_{n m}\right) \hat{B}\right\rangle_{q} .
$$


Rearrange this equation to get

$$
\left\langle\left(\left(e_{q}^{-\beta \hat{E}_{n m}}\right)^{-1}+\left(e_{q}^{-(q-1) \beta \hat{E}_{n m}}\right)\right) \hat{n}_{n m} \hat{B}\right\rangle_{q}=\left\langle\left(e_{q}^{-(q-1) \beta \hat{E}_{n m}}\right) \hat{B}\right\rangle_{q} .
$$

Finally,

$$
\left\langle\hat{n}_{n m} \hat{B}\right\rangle_{q}=\left\langle\frac{\left(e_{q}^{-(q-1) \beta \hat{E}_{n m}}\right)}{\left(\left(e_{q}^{-\beta \hat{E}_{n m}}\right)^{-1}+\left(e_{q}^{-(q-1) \beta \hat{E}_{n m}}\right)\right)} \hat{B}\right\rangle_{q} .
$$

Use the same transformation as in Eqs. (28) and (29). Then Eq. (39) can be written as

$$
\left\langle\hat{n}_{n m} \widehat{\mathrm{B}}\right\rangle_{\mathrm{q}}=\left\langle\frac{1}{\left(\left(e_{q}^{-\beta \hat{E}_{n m}}\right)^{-q}+1\right)} \hat{B}\right\rangle_{q}
$$

and, with Eq. (25), Eq. (40) becomes

$$
\left\langle\hat{n}_{n m} \widehat{\mathrm{B}}\right\rangle_{\mathrm{q}}=\left\langle\frac{1}{\left[1+(q-1) \beta \widehat{E}_{n m}\right]^{\frac{q}{q-1}}+1} \hat{B}\right\rangle_{q} .
$$

It is desirable to write the generating equation (41) as linear in terms of the fluctuations of the local-field operator. This can be done with the aid of the identity [15]

$$
f\left(a+b \Delta \widehat{E}_{n m}\right) \equiv \eta_{0}(n m)+\eta_{1}(n m) \Delta \widehat{E}_{n m}
$$

with

$$
\begin{aligned}
& \eta_{0}(n m) \equiv \frac{1}{2}\left(f\left(a+b \varphi_{n m}\right)+f\left(a-b \varphi_{n m}\right)\right) \\
& \eta_{1}(n m) \equiv \frac{1}{2 \varphi_{n m}}\left(f\left(a+b \varphi_{n m}\right)-f\left(a-b \varphi_{n m}\right)\right) .
\end{aligned}
$$

According to this identity, one can write (40) in the form

$$
\left\langle\hat{n}_{n m} \hat{B}\right\rangle_{q}=\eta_{0}(n m)\langle\hat{B}\rangle_{q}+\eta_{1}(n m)\left\langle\Delta \hat{E}_{n m} \hat{B}\right\rangle_{q},
$$

where

$$
\begin{aligned}
& \eta_{0}(n m) \equiv \frac{1}{2}\left[\frac{1}{\left(\left(e_{q}^{-\beta\left(\left\langle\hat{E}_{n m}\right\rangle-\mu+\varphi_{n m}\right.}\right)^{-q}+1\right)}+\frac{1}{\left(\left(e_{q}^{-\beta\left(\left\langle\hat{E}_{n m}\right\rangle-\mu+\varphi_{n m}\right.}\right)^{-q}+1\right)}\right] \\
& \eta_{1}(n m) \equiv \frac{1}{2 \varphi_{n m}}\left[\frac{1}{\left(\left(e_{q}^{-\beta\left(\left\langle\hat{E}_{n m}\right\rangle-\mu+\varphi_{n m}\right.}\right)^{-q}+1\right)}-\frac{1}{\left(\left(e_{q}^{-\beta\left(\left\langle\hat{E}_{n m}\right\rangle-\mu+\varphi_{n m}\right.}\right)^{-q}+1\right)}\right] .
\end{aligned}
$$

Putting $\hat{B}=1$ in Eq. (45), we obtain the particle distribution:

$$
\left\langle\hat{n}_{n m}\right\rangle=\eta_{0}(n m) \text {. }
$$

Since $\left\langle\Delta \widehat{E}_{n m}\right\rangle=0$, the deviation of the occupation number is defined as

$$
\Delta \hat{n}_{n m}=\hat{n}_{n m}-\left\langle\hat{n}_{n m}\right\rangle \text {. }
$$

Therefore 


$$
\left\langle\Delta \hat{n}_{n m} \hat{B}\right\rangle_{\mathrm{q}}=-\eta_{1}(n m)\left\langle\Delta \widehat{E}_{n m} \hat{B}\right\rangle_{\mathrm{q}}
$$

Again, putting $\hat{B}=\Delta \hat{n}_{n^{\prime} m^{\prime}}$ in Eq. (50), we obtain the pair correlation function:

$$
\left\langle\Delta \hat{n}_{n m} \Delta \hat{n}_{n^{\prime} m^{\prime}}\right\rangle_{\mathrm{q}_{c}}=-\eta_{1}(n m) \sum_{\substack{i j \\ i \neq n^{\prime} \\ j \neq m^{\prime}}} C_{n i m j}\left\langle\Delta \hat{n}_{i j} \Delta \hat{n}_{n^{\prime} m^{\prime}}\right\rangle_{\mathrm{q}}
$$

Here $n \neq n^{\prime}$ and $m \neq m^{\prime}$. The correlation function can be written as [15]

$$
\left\langle\Delta \hat{n}_{n m} \Delta \hat{n}_{n^{\prime} m^{\prime}}\right\rangle_{q}=\left\langle\left(\Delta \hat{n}_{n m}\right)^{2}\right\rangle_{q} \delta_{n n^{\prime}} \delta_{m m^{\prime}}+\left\langle\Delta \hat{n}_{n m} \Delta \hat{n}_{n^{\prime} m^{\prime}}\right\rangle_{q_{c}}
$$

From this equation, the quadratic fluctuations can be expressed in terms of the occupation number:

$$
\left\langle\left(\Delta \hat{n}_{n m}\right)^{2}\right\rangle_{q} \equiv\left\langle\hat{n}^{2}{ }_{n m}\right\rangle_{q}-\left\langle\hat{n}_{n m}\right\rangle_{q}{ }^{2} .
$$

For fermionic systems, $\hat{n}_{n m}^{2}=\hat{n}_{n m}$; so that Eq. (53) becomes

$$
\left\langle\left(\Delta \hat{n}_{n m}\right)^{2}\right\rangle_{q}=\left\langle\hat{n}_{n m}\right\rangle_{q}\left(1-\left\langle\hat{n}_{n m}\right\rangle\right)_{q} .
$$

Finally, putting $\hat{B}=\Delta \widehat{E}_{n m}$ in Eq. (45), we obtain

$$
\eta_{1}(n m) \varphi_{n m}^{2}=\sum_{\substack{i j \\ i \neq n \\ j \neq m}} C_{n i m j}\left\langle\Delta \hat{n}_{n m} \Delta \hat{n}_{i j}\right\rangle_{q_{c}}
$$

We now have a closed system of nonlinear equations for $\left\langle\hat{E}_{n m}\right\rangle_{q},\left\langle\hat{n}_{n m}\right\rangle_{q},\left\langle\Delta \hat{n}_{n m}\right\rangle_{q}$, $\left\langle\Delta \hat{n}_{n m} \Delta \hat{n}_{n^{\prime} m^{\prime}}\right\rangle_{q_{c^{\prime}}}$ and $\varphi_{n m}$. These equations will be solved numerically using Gaussian quadrature.

\section{Thermodynamic Properties of Nanosystems}

Nanosystems are made up of countable numbers of particles in a small 'volume'. Thus, the thermodynamic limit $-N, V \rightarrow \infty ; \frac{N}{V} \rightarrow$ constant - is violated; it follows that conventional thermodynamics and Boltzmann-Gibbs statistical mechanics will not be valid. To compute the thermodynamic properties of these systems, one should first evaluate the grand partition function of a nonextensive system, $Z_{\mathrm{q}}$, defined as $[28,29]$

$$
Z_{q}=\operatorname{Tr}[1-(1-q) \beta(\widehat{H}-\mu N)]^{q / 1-q} .
$$

This can be written as

$$
z_{q}=\operatorname{Tr}\left[e_{q}^{-\beta(\widehat{H}-\mu N)}\right]^{q}
$$




$$
\begin{aligned}
& =\prod_{n m} \sum_{n_{n m}}\left[e_{q}^{-\beta\left(\hat{E}_{n m}-\mu\right) n_{n m}}\right]^{q} \\
& =\prod_{n m}\left[1+\left(e_{q}^{-\beta\left(\hat{E}_{n m}-\mu\right)}\right)^{q}\right] .
\end{aligned}
$$

It is simpler to take the logarithms of both sides:

$$
\begin{aligned}
\ln \left(Z_{q}\right) & =\ln \left(\prod_{n m}\left[1+\left(e_{q}^{-\beta\left(\hat{E}_{n m}-\mu\right)}\right)^{q}\right]\right) \\
& =\sum_{n m} \ln \left[1+\left(e_{q}^{-\beta\left(\hat{E}_{n m}-\mu\right)}\right)^{q}\right] .
\end{aligned}
$$

Using the identity (42), we have

$$
\ln \left(Z_{q}\right)=\sum_{n m} \zeta_{0}(n m)+\zeta_{1}(n m)\left\langle\Delta \hat{E}_{n m}\right\rangle,
$$

where $\zeta_{0}(n m)$ and $\zeta_{1}(n m)$ are given by

$$
\zeta_{0}(n m)=\frac{1}{2} \ln \left[1+\left(e_{q}^{-\beta\left(\left\langle\hat{E}_{n m}\right\rangle-\mu+\varphi_{n m}\right)}\right)^{q}\right]+\ln \left[1+\left(e_{q}^{-\beta\left(\left\langle\hat{E}_{n m}\right\rangle-\mu-\varphi_{n m}\right)}\right)^{q}\right],
$$

and

$$
\zeta_{1}(n m)=\frac{1}{2 \varphi_{n m}} \ln \left[1+\left(e_{q}^{-\beta\left(\left\langle\hat{E}_{n m}\right\rangle-\mu+\varphi_{n m}\right)}\right)^{q}\right]-\ln \left[1+\left(e_{q}^{-\beta\left(\left\langle\hat{E}_{n m}\right\rangle-\mu-\varphi_{n m}\right)}\right)^{q}\right]
$$

The operator $\Delta \hat{E}_{n m}$ is symmetric. Therefore, the second term on the right-hand side of (59) vanishes. Then

$$
\ln \left(Z_{q}\right)=\sum_{n m} \zeta_{0}(n m)
$$

The mean internal energy $\langle\widehat{H}\rangle$ is, then,

$$
\langle\widehat{H}\rangle=-\frac{\partial \ln \left(Z_{q}\right)}{\partial \beta} ;
$$

so that

$$
\begin{aligned}
U= & \frac{1}{2} \sum_{n m} \frac{q\left(\left\langle\hat{E}_{n m}\right\rangle-\mu+\varphi_{n m}\right)\left(e_{q}^{-\beta\left(\left\langle\hat{E}_{n m}\right\rangle-\mu+\varphi_{n m}\right)}\right)^{q-1}\left(e_{q}^{-\beta\left(\left\langle\hat{E}_{n m}\right\rangle-\mu+\varphi_{n m}\right)}\right)^{q}}{\left[1+\left(e_{q}^{-\beta\left(\left\langle\hat{E}_{n m}\right\rangle-\mu+\varphi\right)}\right)^{q}\right]} \\
& +\frac{q\left(\left\langle\hat{E}_{n m}\right\rangle-\mu+\varphi_{n m}\right)\left(e_{q}^{-\beta\left(\left\langle\hat{E}_{n m}\right\rangle-\mu-\varphi_{n m}\right)}\right)^{q-1}\left(e_{q}^{-\beta\left(\left\langle\hat{E}_{n m}\right\rangle-\mu-\varphi_{n m}\right)}\right)^{q}}{\left[1+\left(e_{q}^{-\beta\left(\left\langle\hat{E}_{n m}\right\rangle-\mu-\varphi_{n m}\right)}\right)^{q}\right]}
\end{aligned}
$$




$$
\begin{aligned}
& =\frac{1}{2} \sum_{n m} \frac{q\left(\left\langle\hat{E}_{n m}\right\rangle-\mu+\varphi_{n m}\right)\left(e_{q}^{-\beta\left(\left\langle\hat{E}_{n m}\right\rangle-\mu+\varphi_{n m}\right)}\right)^{2 q-1}}{\left[\left(e_{q}^{-\beta\left(\left\langle\hat{E}_{n m}\right\rangle-\mu+\varphi\right)}\right)^{-q}+1\right]} \\
& +\frac{q\left(\left\langle\hat{E}_{n m}\right\rangle-\mu+\varphi_{n m}\right)\left(e_{q}^{-\beta\left(\left\langle\hat{E}_{n m}\right\rangle-\mu-\varphi_{n m}\right)}\right)^{2 q-1}}{\left[\left(e_{q}^{-\beta\left(\left\langle\hat{E}_{n m}\right\rangle-\mu-\varphi_{n m}\right)}\right)^{-q}+1\right]} .
\end{aligned}
$$

Using the relation (29),

$$
\left(e_{q}^{-\beta\left(\left\langle\hat{E}_{n m}\right\rangle-\mu-\varphi_{n m}\right)}\right)^{2 q-1}=\frac{1}{q}\left(e_{q}^{-\beta\left(\left\langle\hat{E}_{n m}\right\rangle-\mu-\varphi_{n m}\right)}\right)^{q}
$$

we get

$$
U=\frac{1}{2} \sum_{n m} \frac{\left(\left\langle\hat{E}_{n m}\right\rangle+\varphi_{n m}\right)}{\left[\left(e_{q}^{-\beta\left(\left\langle\hat{E}_{n m}\right\rangle-\mu+\varphi_{n m}\right)}\right)^{-q}+1\right]}+\frac{\left(\left\langle\hat{E}_{n m}\right\rangle-\varphi_{n m}\right)}{\left[\left(e_{q}^{-\beta\left(\left\langle\hat{E}_{n m}\right\rangle-\mu-\varphi_{n m}\right)}\right)^{-q}+1\right]},
$$

which is equivalent to

$$
U=\sum_{n m}\left\langle\hat{n}_{n m} \hat{E}_{n m}\right\rangle=\sum_{n m}\left\langle\hat{E}_{n m}\right\rangle\left\langle\hat{n}_{n m}\right\rangle+\varphi_{n m}^{2} \eta_{1}(n m) .
$$

The specific heat at constant volume is evaluated using

$$
\begin{aligned}
C_{v} & =\left(\frac{\partial U}{\partial T}\right)_{A}-\mu\left(\frac{\partial N}{\partial T}\right)_{A} ; \\
C_{v} & =\frac{1}{2} \frac{q}{k_{B} T^{2}} \sum_{n m}\left[\frac{\left(\left\langle E_{n m}\right\rangle-\mu+\varphi_{n m}\right)^{2}\left(e_{q}^{-\beta\left(\left\langle\hat{E}_{n m}\right\rangle-\mu+\varphi_{n m}\right)}\right)^{-1}}{\left(\left(e_{q}^{-\beta\left(\left\langle\hat{E}_{n m}\right\rangle-\mu+\varphi_{n m}\right)}\right)^{-q}+1\right)^{2}}\right. \\
& \left.+\frac{\left(\left\langle E_{n m}\right\rangle-\mu-\varphi_{n m}\right)^{2}\left(e_{q}^{-\beta\left(\left\langle\hat{E}_{n m}\right\rangle-\mu-\varphi_{n m}\right)}\right)^{-1}}{\left(\left(e_{q}^{-\beta\left(\left\langle\hat{E}_{n m}\right\rangle-\mu-\varphi_{n m}\right)}\right)^{-q}+1\right)^{2}}\right] .
\end{aligned}
$$

The entropy can be calculated from

$$
S=\left(U+k_{B} T \ln \left(Z_{q}\right)-\mu N\right) / T .
$$

A word is in order here regarding the values of the quantum numbers $(n, m)$ used in the present calculations. These values were chosen so as to give a 'stable' solution of the coupled equations; i.e., a solution which is independent of $(n, m)$. For more details, see [30].

\section{Results and Discussion}

Graphene is a hexagonal arrangement of carbon atoms. The carbon-carbon bond length in a layer plane is $1.42 \AA$. Carbon atoms are located at the corners of the hexagonal cells. Therefore, the positions of the carbon atoms can be easily determined. The values of $l_{l}$ 
and $l_{2}$ (the dimensions of the 'box') depend on the number of carbon atoms in it as well as on the geometry of the $2 \mathrm{D}$ box. We can calculate $l_{1}$ and $l_{2}$ using properties of regular hexagons. In this work, we studied a rectangular geometry: for $\mathrm{N}=50$ atoms, $l_{1}=29.51 \AA$ and $l_{2}=2.84$ $\AA$; and for $\mathrm{N}=150$ atoms, $l_{1}=91.0 \AA$ and $l_{2}=2.84 \AA$.

The thermodynamic properties of 2D graphene were studied at temperatures around the Fermi degeneracy temperature $T_{F}$ for extensive $(\mathrm{q}=1)$ and nonextensive $(\mathrm{q}=0.9)$ systems. The input potential was the Coulomb interaction. Our results are summarized in Figs.1-9 and Table 1.

Figures 1-3 show the thermodynamic properties of graphene as functions of $T$ for $\mathrm{q}=1$ and 0.9. It is clear that the mean internal energy per electron $U / N$, the specific heat $C_{e l}(T) / N$ and the entropy $S$ increase monotonically with increasing temperature.

In Fig. 1, $U / N$ is plotted as a function of $T$ for different q. It is noted that $U \alpha T$ at $\mathrm{T}>8$ $\mathrm{eV}$. For $T<8 \mathrm{eV}$, the differences between extensive and nonextensive results are not appreciable; while for $T>8 \mathrm{eV}$, the differences become quite tangible.

Figure 2 exhibits our results for $C_{e l}(T) / N$ as functions of $T$. The important point here is that $C_{e l}(T) / N$ varies approximately as the first power of $T$ up to $T=8 \mathrm{eV}$. At $T>8 \mathrm{eV}$, $C_{e l}(T) / N$ goes to the classical limit for the two-dimensional ideal gas $\left(\frac{C_{e l}}{N K_{B}} \rightarrow 1\right)$; while for the nonextensive case it goes to 0.8 instead of 1 .

The behavior of the entropy per unit area $S$ is illustrated in Fig. 3. It is noted that $\mathrm{S}$ goes to zero as $T \rightarrow 0$; which is in agreement with the third law of thermodynamics. Clearly, $S$ increases as q decreases. At $T>20 \mathrm{eV}, S$ is independent of q.

Figures 4 and 5 show the effect of $\mathrm{N}$ on $C_{e l}(T) / N$ and $S / N$. From Fig. 4 , it is clear that $C_{e l}(T) / N$ is independent of $N$; and from Fig. 5, $S / N$ increases as $N$ increases - as expected.

The chemical potential $\mu$ of graphene, shown in Fig. 6, decreases with $T$. The general behavior is consistent with that of an ideal Fermi system. The Fermi degeneracy temperature $\mathrm{T}_{\mathrm{F}} \approx 27 \mathrm{eV}$. The value of $\mathrm{q}$ has almost no effect on $T_{F}$.

Figures 7 and 8 show the mean internal energy per electron $U / N$ and the specific heat $C_{e l}(T) / N$ for 50 atoms of $2 \mathrm{D}$ graphene as functions of $T$ for $T<0.1 \mathrm{eV}$. These quantities have been fitted to the polynomial $\left(T^{\alpha}\right)$. The results are summarized in Table 1 (for $T<0.1 \mathrm{eV}$ ): $C_{e l} \alpha T^{2.07}$ at low $\mathrm{T}$ for extensive systems; while for massless fermions in $2 \mathrm{D}, C_{2 D} \alpha T^{2}$. In a typical 2DEG, $C_{e l} \alpha T$ [11]. Thus, there is equivalence between our model and the masslessfermion-model. Our results indicate that the temperature-dependence of the thermodynamic 
properties of our system decreases with increasing q (nonextensivity). The temperaturedependence of $C_{e l}(T) / N$ at low $T$, calculated using extensive statistical mechanics, is in agreement with [31].Yi et al. [32] investigated low-energy electronic states and the heat capacity of graphene strips. The density of states was obtained by diagonalizing a lattice Hamiltonian for strips with zigzag and armchair edges, respectively. It was found that the overall behavior of the heat capacity $C_{e l}$ was similar to that of a two-level system, and the low-temperature heat capacity showed unconventional behavior of $C_{e l} \alpha T^{\alpha}$ with $\alpha \approx 2.2$, but varying slightly for different choices of band-structure parameters and strip width.

Figure 9 represents the equation of state $f_{L} A=k_{B} T \ln Z_{q}$ of our system as a function of $T$ for $\mathrm{q}=1$ and $0.9\left(f_{L}\right.$ being the force per unit length). It is noted that $f_{L} A$ for the nonextensive case is higher than that for the extensive case. At low $T, f_{L} A$ increases monotonically in the extensive case; while it increases dramatically in the nonextensive case. At high $T$, this quantity increases monotonically in both extensive and nonextensive cases.

\section{Conclusion}

It was found that, for the present system, the mean internal energy per electron $U / N$, the specific heat $C_{e l}(T) / N$ and the entropy $S$ increase with increasing temperature. Also, it was established that these properties are less sensitive to the temperature when using nonextensive - in contrast to extensive - statistical mechanics. In addition, it was found that $C_{e l}(T) / N$ is independent of $N$, and $S / N$ increases as $N$ increases - as expected. It was noted that the mean internal energy and the specific heat behave as a power-law $T^{\alpha}$ at $T<8 \mathrm{eV}$; whereas they go to the classical limit for the two-dimensional ideal gas at $T>8 \mathrm{eV}$. The general behavior of the chemical potential is consistent with that of an ideal Fermi system.

In future work, it is intended to calculate the thermodynamic properties of graphene using the relativistic Dirac equation, instead of the nonrelativistic Schrödinger equation. This means that SFA for the relativistic case should first be derived and studied. 


\section{References}

[1] J. C.Charlier, X. Blasé, and S. Roche, Reviews of Modern Physics, 79 (2007) 677.

[2] M. Ezawa, Physica E 40, (2008) 1421.

[3] G. Grushin, B. Valenzuela, and M. Vozmediano, Physical Review B, 80 (2009) 155417.

[4] K. S. Novoselov, A. K. Geim, S. V. Morozov, D. Jiang, M. I. Katsnelson, I. V. Grigorieva, S. V. Dubonos, and A. A. Firsov, Nature, 438 (2005) 197.

[5] A. K. Geim, Science, 324 (2009)1531.

[6] Y. Zhang, Y. W. Tan, L. Stormer, and P. Kim, Nature, 438 (2005) 201.

[7] A. V. Markin, N. N. Smirnova, I. E. Boronina, V. A. Ruchenin, and A. G. Lyapin, Russian Chemical Bulletin, International Edition, 57 (2008) 1975.

[8] A. R. Wright, Junfeng Liu, Zhongshui Ma, Z. Zeng, W. Xu, and C. Zhang, Microelectronics Journal, 40 (2008) 716.

[9] R. Nasir, M. A. Khan, M. Tahir, and K. Sabeeh, Journal of Physics: Condensed Matter, 22 (2010) 25503.

[10] M. K. Li, S. J. Lee, and T. W. Kang, Current Applied Physics, 9 (2009) 769.

[11] K. S. Yi, D. Kim, K. S. Park, and, J. J. Quinn, Physica E, 40 (2008) 1715.

[12] R. R. Nigmatullin, and V. A. Toboev, Theoretical and Mathematical Physics, 80 (1989) 94.

[13] R. R. Nigmatullin, A. A. Khamzin, and H. B. Ghassib, Physical Review E, 61 (2000) 3441.

[14] M. K. Al-Sugheir, H. B. Ghassib, and R. R. Nigmatullin, International Journal of Theoretical Physics, 40 (2001) 1033.

[15] M. K. Al-Sugheir, and H. B. Ghassib, International Journal of Theoretical Physics, 41 (2002) 705 .

[16] R. R. Nigmatullin, A. A. Khamzin, and I. I. Popov, Journal of Experimental and Theoretical Physics JETP, 114 (2012) 312.

[17] B. R. Joudeh, M. K. Al-Sugheir, and H. B. Ghassib, International Journal of Modern Physics B, 19 (2005) 3985.

[18] A. S. Sandouqa, M. K. Al-Sugheir, and H. B. Ghassib, International Journal of Theoretical Physics, 45 (2006)159.

[19] N. M. Ghulam, H.B. Ghassib, and M. K. Al-Sugheir, Physical Review C, 75(2007) 064317. 
[20] S. I. Qashou, M. K. Al-Sugheir, A. R. Sakhel, and H. B. Ghassib, International Journal of Modern Physics B, 24 (2010) 4779.

[21] P. Mohazzabi and A. Mansoori, Journal of Computational and Theoretical Nanoscience, 2 (2005) 1.

[22] Ph. Chomaz, V. Duflot,and F. Gulminelli, Physical Review Letters, 85 (2000) 3587.

[23] C. Tsallis, Journal of Statistical Physics, 52 (1988) 479.

[24] T. L. Hill, Thermodynamics of Small Systems (Dover, New York, 1964).

[25] A. M. Nassimi and G. A. Parsafar, Journal of Physics: Condensed Matter, 19 (2007) 156218.

[26] Fetter, A. L. and Walecka, J. D. Quantum Theory of Many-Particle System, McGrawHill, New York, 1971.

[27] Feynman, R. P. Statistical Mechanics, Benjamin, Reading, MA (1992).

[28] Q. A. Wang and A. L. Mehaut, Journal of Mathematical Physics, 43 (2002) 5079.

[29] Q. A. Wang, The European Physical Journal B, 26 (2002) 357.

[30] M. K. Al-Sugheir, G.Alna'washi, H.B.Ghassib, and A.Sandouqa, Physica B, 407 (2012) 2313.

[31] K. S. Yi, D. Kim, K. S. Park, and S. Kim, Journal of the Korean Physical Society, 50 (2007)1674.

[32] K. S. Yi, D. Kim and K. S. Park, Physical Review B, 76, (2007)115410. 


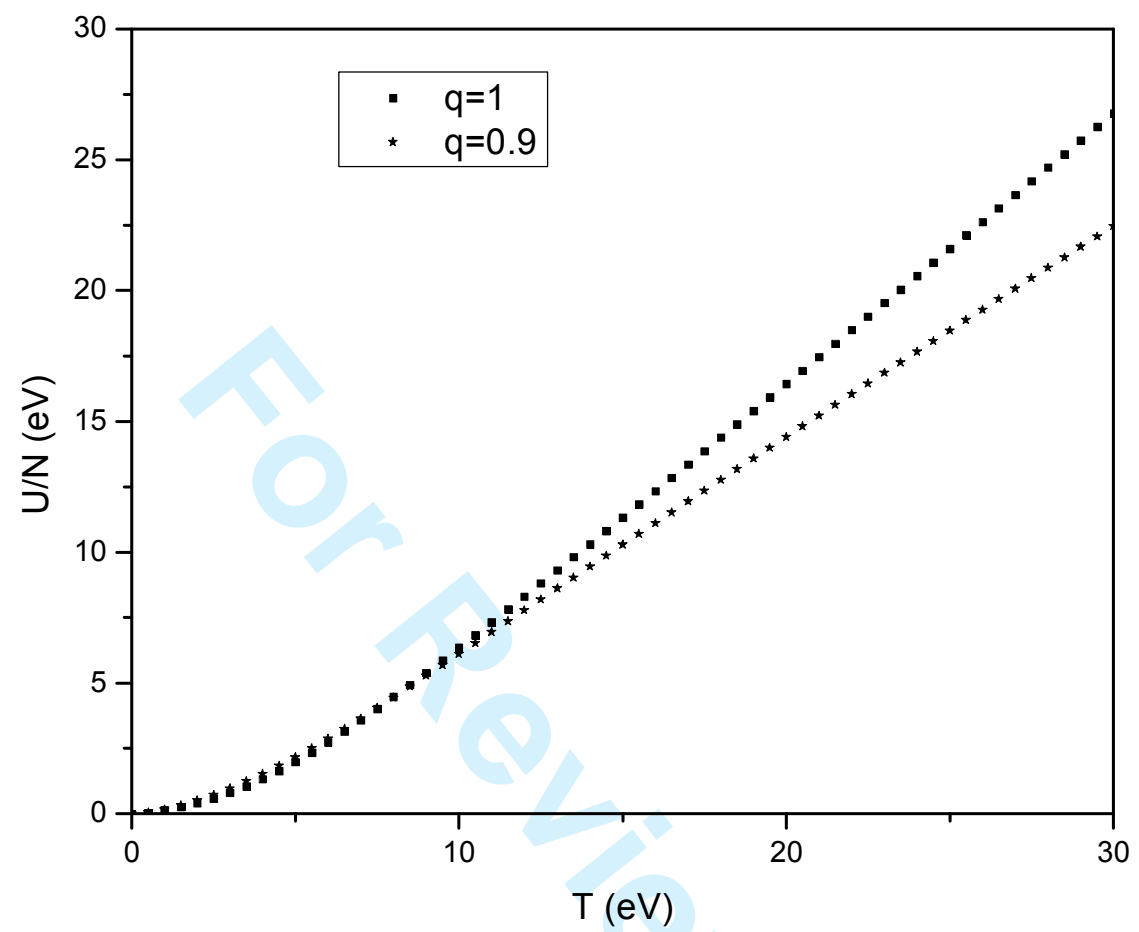

Figure 1: The temperature-dependence of the internal energy per atom $U / N$, for extensive ( $\mathrm{q}=1)$ and nonextensive $(\mathrm{q}=0.9)$ statistical mechanics; $N=50$ atoms. 


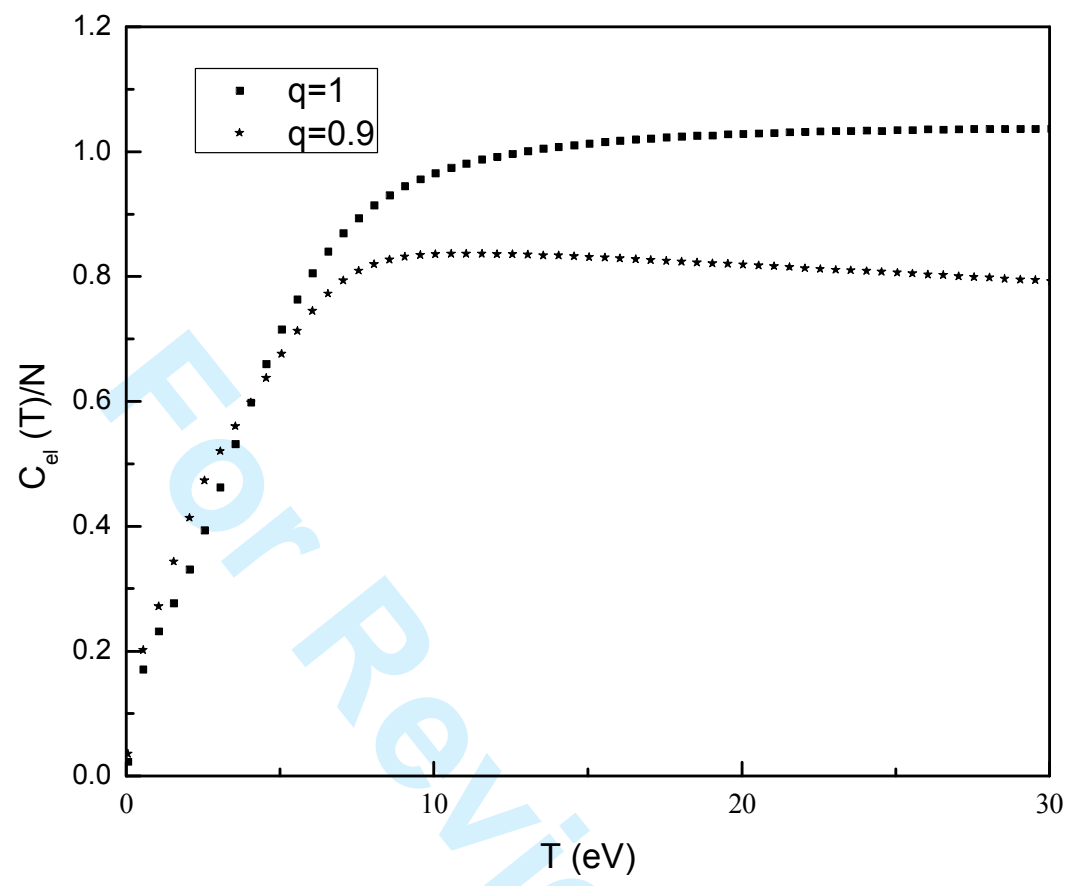

Figure 2: The temperature-dependence of the specific heat $\frac{C_{e l}(T)}{N}$, for extensive $(\mathrm{q}=1)$ and nonextensive (q=0.9) statistical mechanics; $N=50$ atoms. 


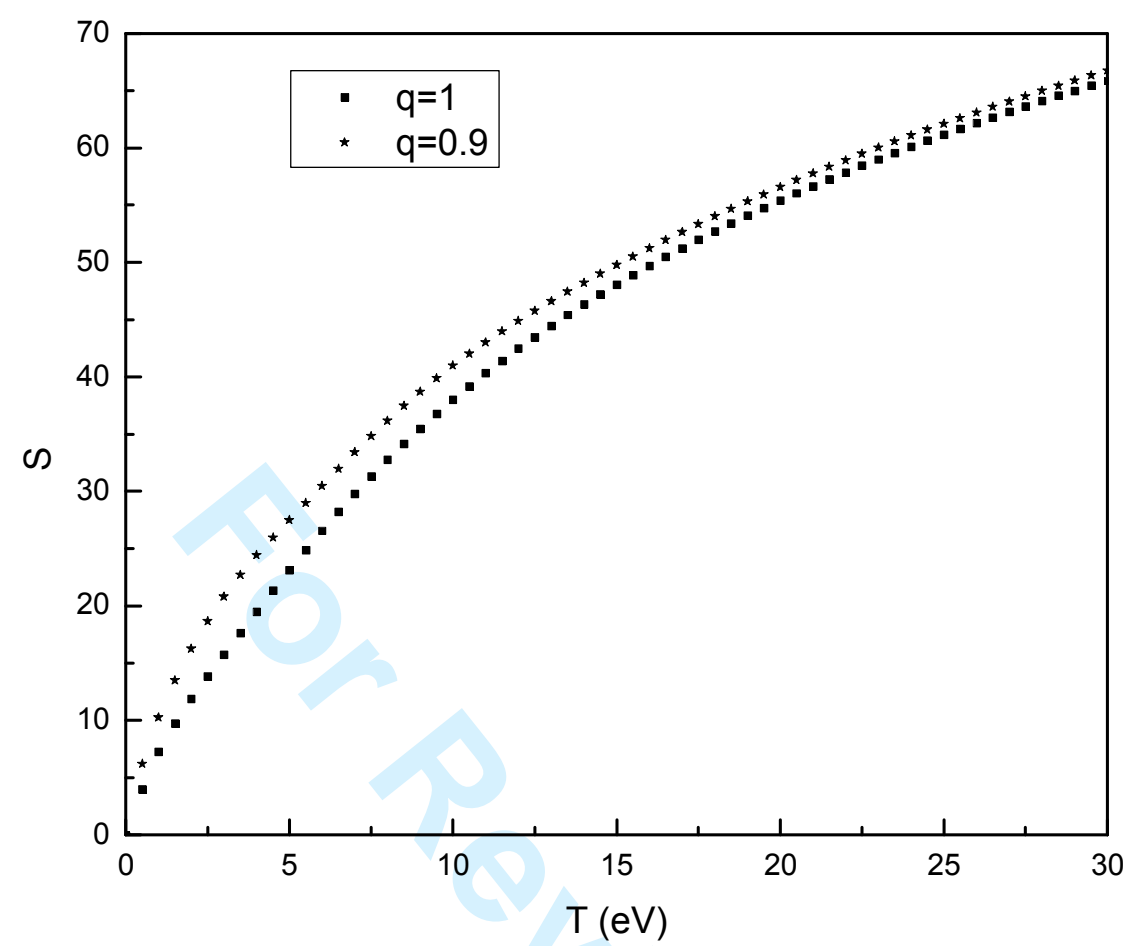

Figure 3: The temperature-dependence he entropy $S$, for extensive $(\mathrm{q}=1)$ and nonextensive (q=0.9) statistical mechanics; $N=50$ atoms. 


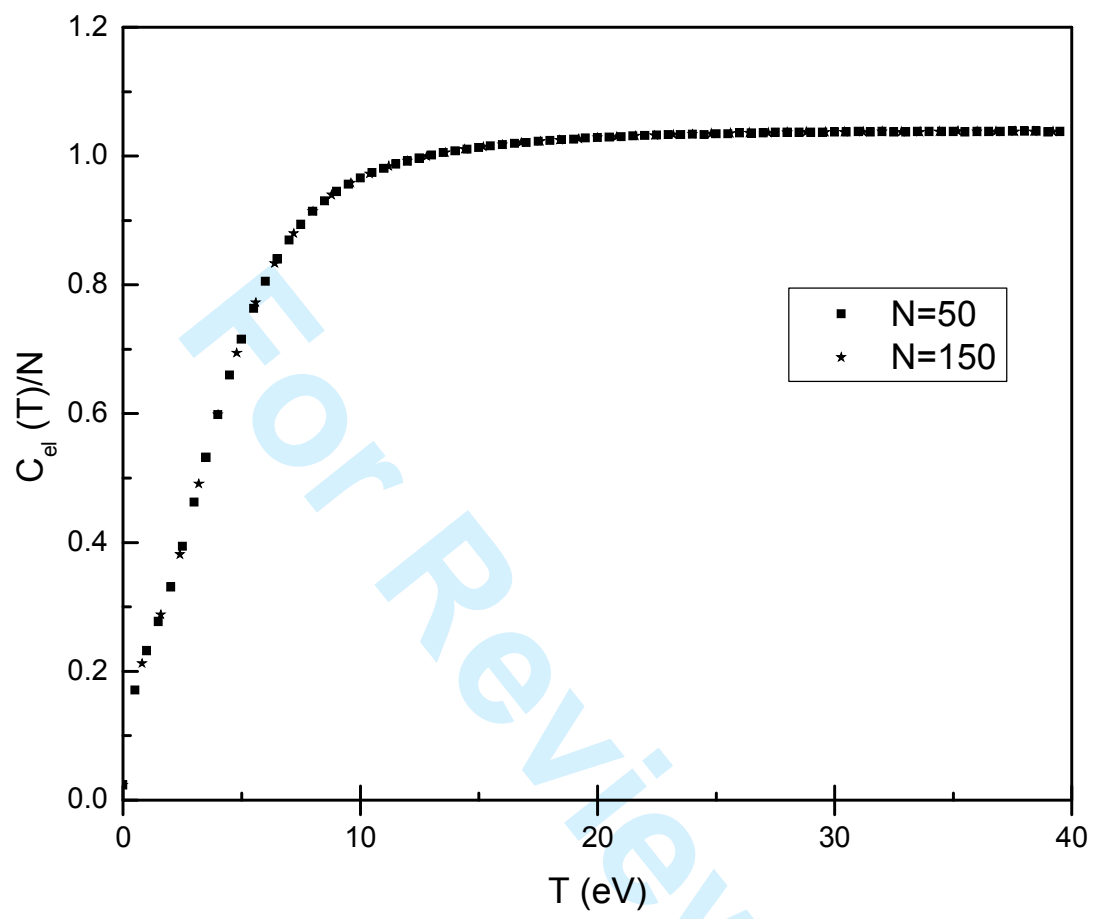

Figure 4: The temperature-dependence of the specific heat $\frac{C_{e l}(T)}{N}$, for extensive $(\mathrm{q}=1)$ statistical mechanics; $N=50$ and 150 atoms. 


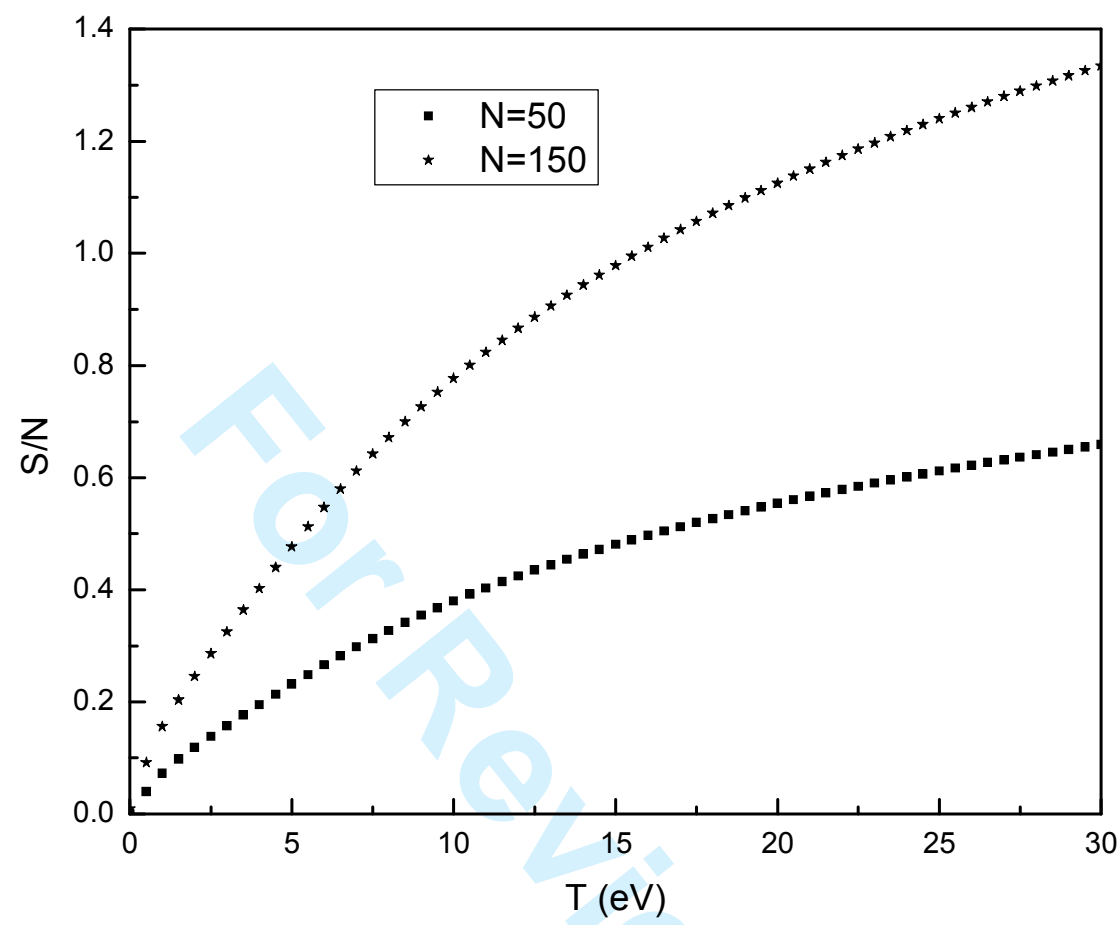

Figure 5: The temperature-dependence of the entropy $S$ for extensive (q=1) statistical mechanics; $N=50$ and 150 atoms. 


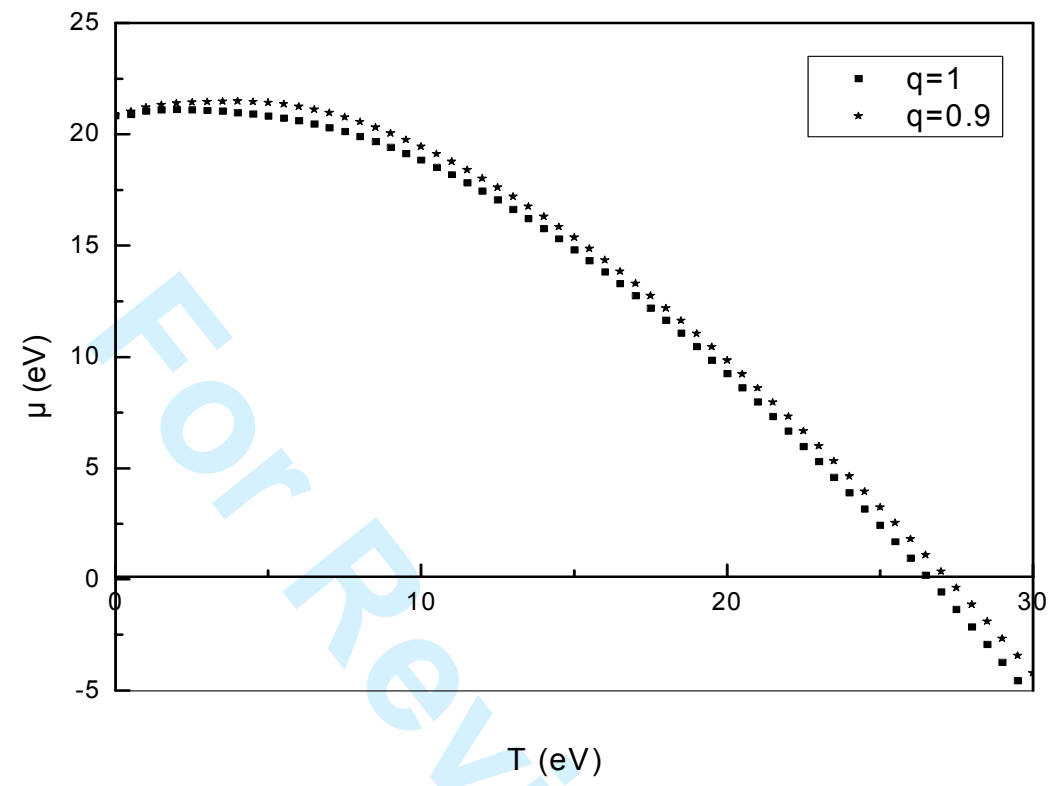

Figure 6: The temperature-dependence of the 'effective' chemical potential $\mu$, for extensive $(\mathrm{q}=1)$ and nonextensive $(\mathrm{q}=0.9)$ statistical mechanics; $N=50$ atoms. 


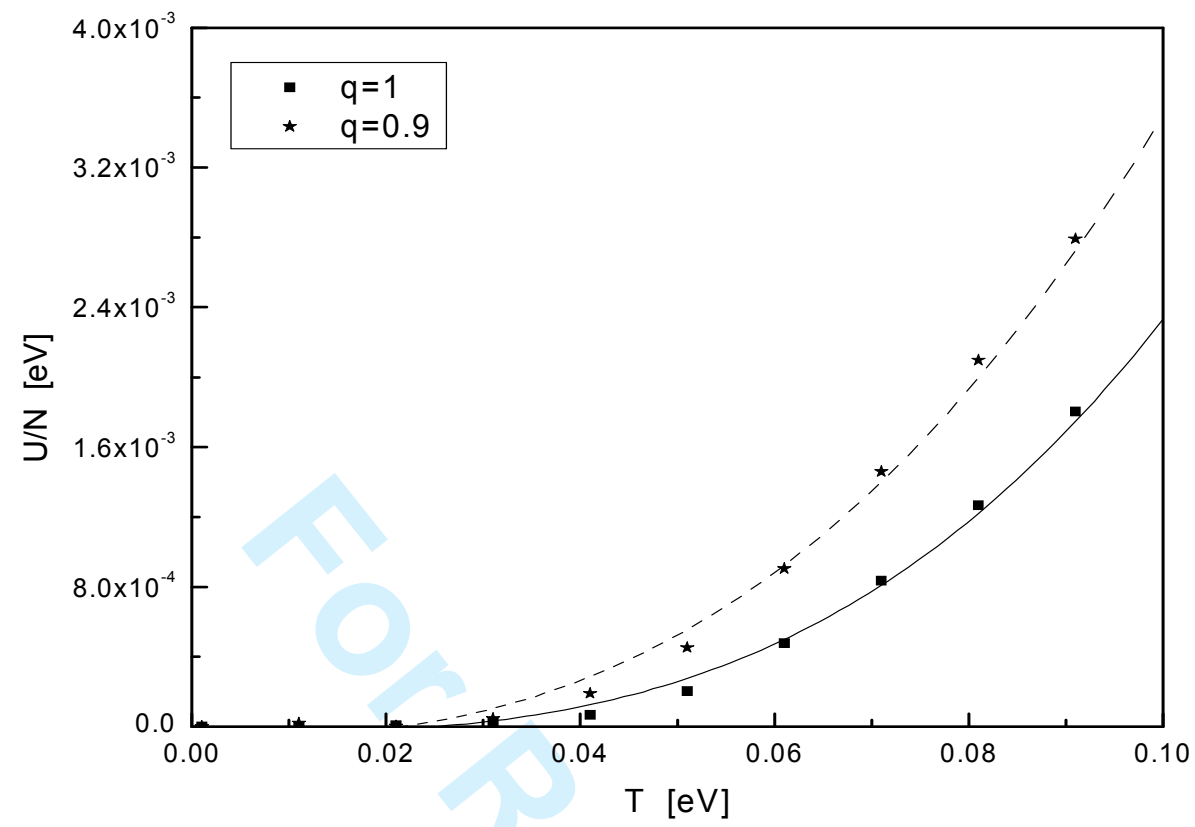

Figure 7: The temperature-dependence of the internal energy per atom $U / N$, for extensive and nonextensive statistical mechanics; $N=50$ atoms and $T<0.1 \mathrm{eV}$. The dashed line indicates the fit of nonextensive data; whereas the solid line pertains to the fit of extensive data. 


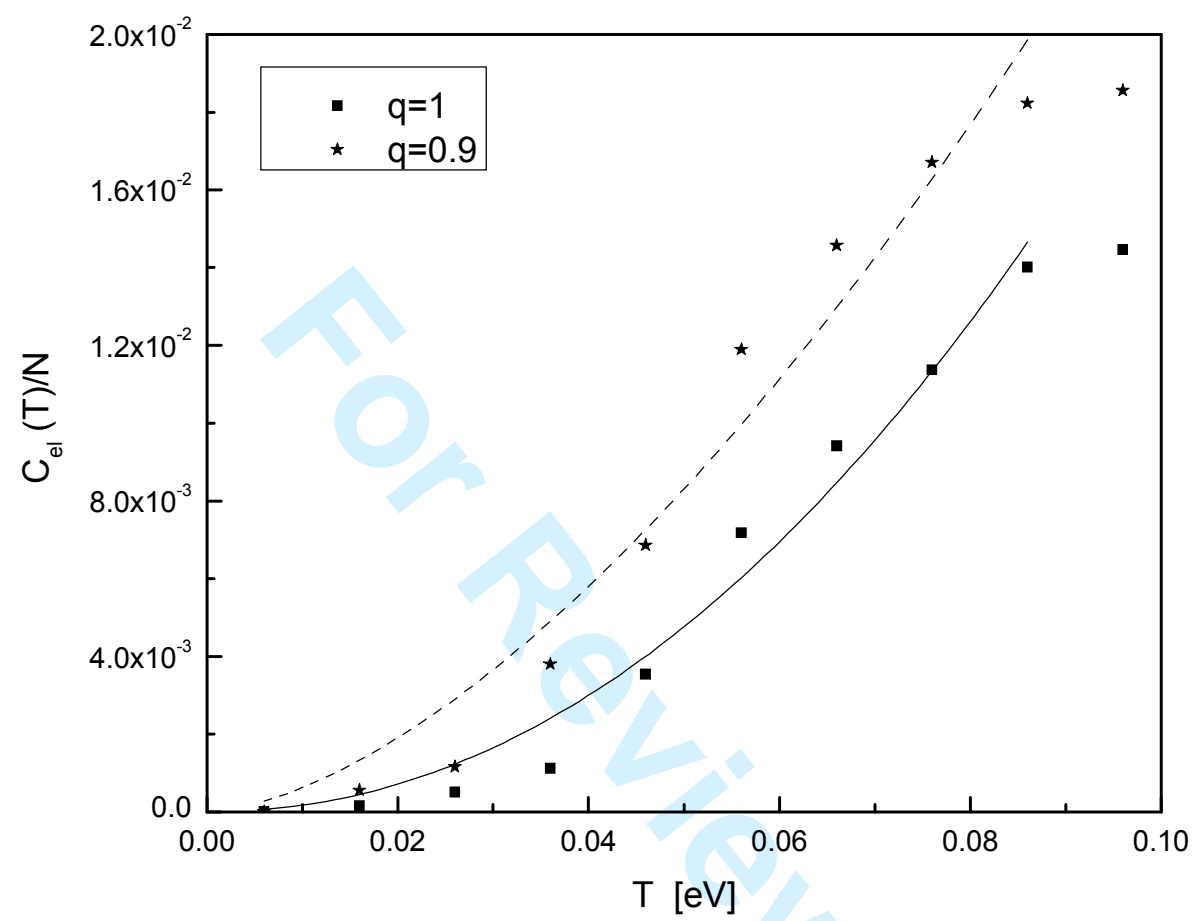

Figure 8: The temperature-dependence of the heat capacity $C_{e l}(T)$, for extensive and nonextensive statistical mechanics; $N=50$ atoms and $T<0.1 \mathrm{eV}$. The dashed line indicates the fit of nonextensive data; whereas the solid line pertains to the fit of extensive data. 


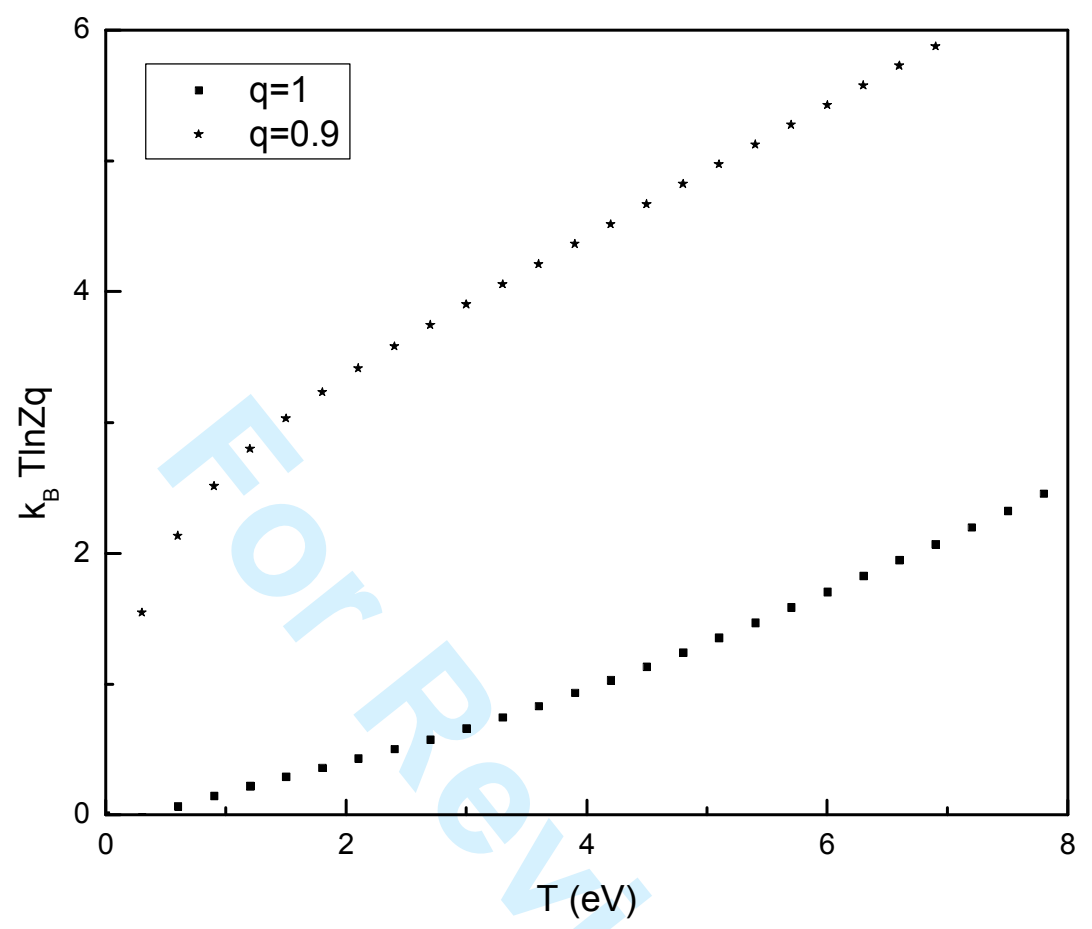

Figure 9: The equation of state for both extensive and nonextensive statistical mechanics for $T$ up to $8 \mathrm{eV} ; N=50$ atoms. 
Table 1: The parameter $\alpha$ for extensive ( $\mathrm{q}=1)$ and nonextensive $(\mathrm{q}=0.9)$ systems; $T<0.1 \mathrm{eV}$.

\begin{tabular}{|c|c|c|}
\cline { 2 - 3 } \multicolumn{1}{c|}{} & \multicolumn{2}{|c|}{$\alpha$} \\
\hline Entropy parameter & $\mathrm{q}=1$ & $\mathrm{q}=0.9$ \\
\hline$U / N \quad(\mathrm{eV})$ & 3.01 & 2.59 \\
\hline$C_{e l}$ & 2.07 & \\
& {$[2 \sim 3.5]^{\mathrm{a}}$} & 1.61 \\
{$[2.27]^{\mathrm{b}}$} & \\
& {$[2.5 \text { and } 2.7]^{\mathrm{c}}$} & \\
{$[2.2]^{\mathrm{d}}$} & \\
\hline
\end{tabular}

${ }^{\mathrm{a}}[31]$

$\mathrm{b}[11]$

[ [7]

d [32] 